\title{
Optimal bounds for the sine and hyperbolic tangent means IV
}

\section{Monika Nowicka ${ }^{1}$ (D) Alfred Witkowski ${ }^{2}$}

Received: 6 October 2020 / Accepted: 9 March 2021 / Published online: 18 March 2021

(c) The Author(s) 2021

\section{Abstract}

We provide optimal bounds for the sine and hyperbolic tangent means in terms of various weighted means of the arithmetic and centroidal means

Keywords Sine mean $\cdot$ Hyperbolic tangent mean $\cdot$ Seiffert function

Mathematics Subject Classification 26D15

\section{Introduction, definitions and notations}

The means

$$
\mathrm{M}_{\sin }(x, y)= \begin{cases}\frac{x-y}{2 \sin \frac{x-y}{x+y}} & x \neq y \\ x & x=y\end{cases}
$$

and

$$
\mathrm{M}_{\tanh }(x, y)= \begin{cases}\frac{x-y}{2 \tanh \frac{x-y}{x+y}} & x \neq y \\ x & x=y\end{cases}
$$

defined for positive arguments, have been introduced in [19], where one of the authors investigates means of the form

$$
\mathrm{M}_{f}(x, y)=\left\{\begin{array}{ll}
\frac{|x-y|}{2 f\left(\frac{|x-y|}{x+y}\right)} & x \neq y \\
x & x=y
\end{array} .\right.
$$

Monika Nowicka

monika.nowicka@utp.edu.pl

Alfred Witkowski

audomat.aw@gmail.com

1 Institute of Mathematics and Physics, UTP University of Science and Technology, al. prof.

Kaliskiego 7, 85-796 Bydgoszcz, Poland

2 AUDoMAT, ul. Mielczarskiego 4/29, 85-796 Bydgoszcz, Poland 
It was shown that every symmetric and homogeneous mean of positive arguments can be represented in the form (1) and that every function $f:(0,1) \rightarrow \mathbb{R}$ (called Seiffert function) satisfying

$$
\frac{z}{1+z} \leq f(z) \leq \frac{z}{1-z}
$$

produces a mean. The correspondence between means and Seiffert functions is given by the formula

$$
f(z)=\frac{z}{M(1-z, 1+z)}, \text { where } z=\frac{|x-y|}{x+y} .
$$

Comparing the means and examining the relationships between them is considered important. A cursory overview of MathSciNet shows over 200 papers on this subject, and the number is constantly growing. The aim of this paper is to determine various optimal bounds for the $M_{\tanh }$ and $M_{\text {sin }}$ with the arithmetic and centroidal means (denoted here by $A$ and Ce). Similar bounds by the arithmetic and contraharmonic means were obtained in [12], and by arithmetic and quadratic means in [11]. For other bounds of Seiffert-like means by the arithmetic and centroidal means, see e.g. $[7,8,17,20]$. Similar subjects were considered also in $[2-6,10,13-16,18,21]$.

For two means $M, N$, the symbol $M<N$ denotes that for all positive $x \neq y$ the inequality $M(x, y)<N(x, y)$ holds.

Our main tool will be the obvious fact that if for two Seiffert functions the inequality $f<g$ holds, then their corresponding means satisfy $M_{f}>M_{g}$. Thus every inequality between means can be replaced by the inequality between their Seiffert functions.

Remark 1 Throughout this paper all means are defined on $(0, \infty)^{2}$.

Remark 2 Note that the Seiffert function of the centroidal mean $\operatorname{Ce}(x, y)=\frac{2}{3} \frac{x^{2}+x y+y^{2}}{x+y}$ is $\operatorname{ce}(z)=\frac{3 z}{3+z^{2}}$ and that of the arithmetic mean $\mathrm{A}(x, y)=\frac{x+y}{2}$ is the identity function $\mathrm{a}(z)=z$. Clearly, the Seiffert functions of $M_{\sin }$ and $M_{\tanh }$ are the functions sin and tanh, respectively.

For the reader's convenience, in the following sections we place the main results with their proofs, while all lemmas and technical details can be found in the last section of this paper.

The motivation for our research are the inequalities $A<M_{\text {sin }}<M_{\tanh }<$ Ce proven in [19, Lemma 3.1] and Lemma 1.

\section{Linear bounds}

Given three means $K<L<M$, one may try to find the best $\alpha, \beta$ satisfying the double inequality $(1-\alpha) K+\alpha M<L<(1-\beta) K+\beta M$ or equivalently $\alpha<\frac{L-K}{M-K}<\beta$. If $k, l, m$ are respective Seiffert functions, then the latter can be written as

$$
\alpha<\frac{\frac{1}{l}-\frac{1}{k}}{\frac{1}{m}-\frac{1}{k}}<\beta .
$$

Therefore the problem reduces to finding upper and lower bounds for certain functions defined on the interval $(0,1)$. 
Theorem 1 The inequalities

$$
(1-\alpha) A+\alpha C e<M_{\sin }<(1-\beta) A+\beta C e
$$

hold if, and only if, $\alpha \leq \frac{1}{2}$ and $\beta \geq \frac{3}{\sin 1}-3 \approx 0.5652$.

Proof By formula (2) and Remark 2, we investigate the function

$$
h(z)=\frac{\frac{1}{\sin z}-\frac{1}{z}}{\frac{3+z^{2}}{3 z}-\frac{1}{z}}=\frac{3}{z \sin z}-\frac{3}{z^{2}} .
$$

We shall show that $h$ increases. Observe that

$$
h^{\prime}(z)=3 \frac{2 \sin ^{2} z-z^{2} \cos z-z \sin z}{z^{3} \sin ^{2} z}=: 3 \frac{s(z)}{z^{3} \sin ^{2} z} .
$$

Using the known inequalities $x-x^{3} / 3 !<\sin x<x-x^{3} / 3 !+x^{5} / 5$ ! and $\cos x<1-$ $x^{2} / 2$ ! $+x^{4} / 4$ ! we get

$$
s(z)>2\left(z-\frac{z^{3}}{3 !}\right)^{2}-z^{2}\left(1-\frac{z^{2}}{2 !}+\frac{z^{4}}{4 !}\right)-z\left(z-\frac{z^{3}}{3 !}+\frac{z^{5}}{5 !}\right)=\frac{z^{6}}{180}>0,
$$

so $h^{\prime}(z)>0$. We complete the proof by noting that $\lim _{z \rightarrow 0} h(z)=1 / 2$.

Theorem 2 The inequalities

$$
(1-\alpha) A+\alpha C e<M_{\tanh }<(1-\beta) A+\beta C e
$$

hold if, and only if, $\alpha \leq \frac{3}{\tanh 1}-3 \approx 0.9391$ and $\beta \geq 1$.

Proof We use Remark 2 and formula (2) once more and investigate the function

$$
h(z)=\frac{\frac{1}{\tanh z}-\frac{1}{z}}{\frac{1+z^{2} / 3}{z}-\frac{1}{z}}=\frac{3}{z}\left(\frac{1}{\tanh z}-\frac{1}{z}\right)=: \frac{3 s(z)}{z} .
$$

The function $s$ satisfies $\lim _{z \rightarrow 0} s(z)=0$ and $s^{\prime \prime}(z)=\frac{2}{\sinh ^{3} z}\left(\cosh z-\frac{\sinh ^{3} z}{z^{3}}\right)<0$ (by Lemma 2), so $s$ is concave and, by Property 2, its divided difference (and consequently the function $h$ ) decreases. To complete the proof note that $\lim _{z \rightarrow 0} h(z)=1$.

\section{Harmonic bounds}

In this section, we look for optimal bounds for means $K<L<M$ of the form $\frac{1-\alpha}{M}+\frac{\alpha}{K}<$ $\frac{1}{L}<\frac{1-\beta}{M}+\frac{\beta}{K}$ or, in terms of their Seiffert functions,

$$
\alpha<\frac{l-m}{k-m}<\beta .
$$

We shall use the above to prove two theorems.

Theorem 3 The inequalities

$$
\frac{1-\alpha}{C e}+\frac{\alpha}{A}<\frac{1}{M_{\sin }}<\frac{1-\beta}{C e}+\frac{\beta}{A}
$$

hold if, and only if, $\alpha \leq 4 \sin 1-3 \approx 0.3659$ and $\beta \geq \frac{1}{2}$. 
Proof According to formula (3), we investigate the function

$$
h(z)=\frac{\sin z-\frac{3 z}{z^{2}+3}}{z-\frac{3 z}{z^{2}+3}}=\frac{\left(z^{2}+3\right) \sin z-3 z}{z^{3}} .
$$

We shall show that $h$ decreases. We have

$$
h^{\prime}(z)=\frac{\left(z^{3}+3 z\right) \cos z-\left(z^{2}+9\right) \sin z+6 z}{z^{4}}=: \frac{s(z)}{z^{4}} .
$$

The function $s$ satisfies $s(0)=s^{\prime}(0)=s^{\prime \prime}(0)=0$ and

$$
s^{\prime \prime \prime}(z)=z\left(\left(z^{2}-9\right) \sin z-8 z \cos z\right)<0 .
$$

Thus $s$ is negative and so is $h^{\prime}$. We complete the proof by noting that $\lim _{z \rightarrow 0} h(z)=1 / 2$.

Theorem 4 The inequalities

$$
\frac{1-\alpha}{\mathrm{Ce}}+\frac{\alpha}{A}<\frac{1}{M_{\tanh }}<\frac{1-\beta}{\mathrm{Ce}}+\frac{\beta}{A}
$$

hold if, and only if, $\alpha \leq 0$ and $\beta \geq 4 \tanh 1-3 \approx 0.0464$.

Proof We use Remark 2 and formula (3) once more and investigate the function

$$
h(z)=\frac{\tanh z-\frac{3 z}{3+z^{2}}}{z-\frac{3 z}{3+z^{2}}}=\frac{\left(3+z^{2}\right) \tanh z-3 z}{z^{3}} .
$$

We shall show that $h$ increases. We have

$$
\begin{aligned}
h^{\prime}(z) & =\frac{6 z+z\left(3+z^{2}\right) \frac{1}{\cosh ^{2} z}-\left(9+z^{2}\right) \frac{\sinh z}{\cosh z}}{z^{4}} \\
& =\frac{12 z+2 z^{3}+6 z \cosh 2 z-\left(9+z^{2}\right) \sinh 2 z}{2 z^{4} \cosh ^{2} z}=: \frac{s(z)}{2 z^{4} \cosh ^{2} z} .
\end{aligned}
$$

By Lemma 4 we get

$$
\begin{aligned}
s(z) & >12 z+2 z^{3}+6 z\left(1+\frac{(2 z)^{2}}{2 !}+\frac{(2 z)^{4}}{4 !}+\frac{(2 z)^{6}}{6 !}\right) \\
& -\left(9+z^{2}\right)\left(2 z+\frac{(2 z)^{3}}{3 !}+\frac{(2 z)^{5}}{5 !}+2 \frac{(2 z)^{7}}{7 !}\right)=\frac{4 z^{5}}{315}\left(21-15 z^{2}-4 z^{4}\right)>0 .
\end{aligned}
$$

Therefore $h$ increases from $\lim _{z \rightarrow 0} h(z)=0$ to $h(1)$.

\section{Quadratic bounds}

Given three means $K<L<M$, one may try to find the best $\alpha, \beta$ satisfying the double inequality $\sqrt{(1-\alpha) K^{2}+\alpha M^{2}}<L<\sqrt{(1-\beta) K^{2}+\beta M^{2}}$ or equivalently $\alpha<\frac{L^{2}-K^{2}}{M^{2}-K^{2}}<\beta$. If $k, l, m$ are respective Seiffert functions, then the latter can be written as

$$
\alpha<\frac{\frac{1}{l^{2}}-\frac{1}{k^{2}}}{\frac{1}{m^{2}}-\frac{1}{k^{2}}}<\beta .
$$


Thus, the problem reduces to finding upper and lower bounds for certain functions defined on the interval $(0,1)$.

Theorem 5 The inequalities

$$
\sqrt{(1-\alpha) A^{2}+\alpha C e^{2}}<M_{\sin }<\sqrt{(1-\beta) A^{2}+\beta C e^{2}}
$$

hold if, and only if, $\alpha \leq \frac{1}{2}$ and $\beta \geq \frac{9}{7} \cot ^{2} 1 \approx 0.5301$.

Proof Using formula (4) we investigate the function

$$
h(z)=\frac{\frac{1}{\sin ^{2} z}-\frac{1}{z^{2}}}{\frac{\left(z^{2}+3\right)^{2}}{9 z^{2}}-\frac{1}{z^{2}}}=\frac{\frac{z^{2}}{\sin ^{2} z}-1}{\frac{\left(z^{2}+3\right)^{2}}{9}-1} .
$$

To show that $h$ increases we use Lemma 3. A simple calculation shows that

$$
r(z)=\frac{\left(\frac{z^{2}}{\sin ^{2} z}-1\right)^{\prime}}{\left(\frac{\left(z^{2}+3\right)^{2}}{9}-1\right)^{\prime}}=\frac{9(\sin z-z \cos z)}{2\left(z^{2}+3\right) \sin ^{3} z}
$$

and

$$
r^{\prime}(z)=9 \frac{2\left(2 z^{3}+5 z\right)-\left(z^{2}+9\right) \sin 2 z+2 z\left(z^{2}+4\right) \cos 2 z}{4\left(z^{2}+3\right)^{2} \sin ^{4} z}=: 9 \frac{s(z)}{4\left(z^{2}+3\right)^{2} \sin ^{4} z} .
$$

From $\sin 2 x<2 x-(2 x)^{3} / 3 !+(2 x)^{5} / 5$ ! and $\cos 2 x>1-(2 x)^{2} / 2 !+(2 x)^{4} / 4 !-(2 x)^{6} / 6$ ! we get

$$
\begin{aligned}
s(z)> & 2\left(2 z^{3}+5 z\right)-\left(z^{2}+9\right)\left(2 z-\frac{(2 z)^{3}}{3 !}+\frac{(2 z)^{5}}{5 !}\right) \\
& +2 z\left(z^{2}+4\right)\left(1-\frac{(2 z)^{2}}{2 !}+\frac{(2 z)^{4}}{4 !}-\frac{(2 z)^{6}}{6 !}\right) \\
= & \frac{4}{45} z^{5}\left(-2 z^{4}+4 z^{2}+3\right)>0 .
\end{aligned}
$$

Thus $r^{\prime}$ is positive and both $r$ and $h$ increase. We complete the proof by noting that $\lim _{z \rightarrow 0} h(z)=1 / 2$.

And here comes the hyperbolic tangent version of the previous theorem.

Theorem 6 The inequalities

$$
\sqrt{(1-\alpha) A^{2}+\alpha C e^{2}}<M_{\tanh }<\sqrt{(1-\beta) A^{2}+\beta C e^{2}}
$$

hold if, and only if, $\alpha \leq \frac{9}{7}\left(\operatorname{coth}^{2} 1-1\right) \approx 0.9309$ and $\beta \geq 1$.

Proof We shall use the identity $\tanh ^{2} z=\frac{\cosh 2 z-1}{\cosh 2 z+1}$.

The function to be considered here is

$$
h(z)=\frac{\frac{1}{\tanh ^{2} z}-\frac{1}{z^{2}}}{\frac{\left(z^{2}+3\right)^{2}}{9 z^{2}}-\frac{1}{z^{2}}}=9 \frac{1+z^{2}+\left(z^{2}-1\right) \cosh 2 z}{\left(z^{4}+6 z^{2}\right)(\cosh 2 z-1)},
$$


and its derivative equals

$$
h^{\prime}(z)=-\frac{18 z \sinh z}{\left(z^{4}+6 z^{2}\right)^{2}(\cosh 2 z-1)^{2}} s(z),
$$

where

$$
s(z)=4 z^{3}\left(z^{2}+6\right) \cosh z+\left(z^{4}+6 z^{2}+18\right) \sinh z-\left(6+2 z^{2}-z^{4}\right) \sinh 3 z
$$

and by Lemma 4

$$
\begin{gathered}
s(z)>\left(\begin{array}{c}
4 z^{3}\left(z^{2}+6\right)\left(1+\frac{z^{2}}{2 !}+\frac{z^{4}}{4 !}+\frac{z^{6}}{6 !}+\frac{z^{8}}{8 !}\right) \\
+\left(z^{4}+6 z^{2}+18\right)\left(z+\frac{z^{3}}{3 !}+\frac{z^{5}}{5 !}+\frac{z^{7}}{7 !}\right) \\
-\left(6+2 z^{2}-z^{4}\right)\left(3 z+\frac{(3 z)^{3}}{3 !}+\frac{(3 z)^{5}}{5 !}+\frac{(3 z)^{7}}{7 !}+2 \frac{(3 z)^{9}}{9 !}\right)
\end{array}\right) \\
=z^{7} \frac{2189 z^{6}+4502 z^{4}+14430 z^{2}+21504}{20160}>0 .
\end{gathered}
$$

This shows that $h^{\prime}<0$ so $h$ decreases from $\lim _{z \rightarrow 0} h(z)=1$ to $h(1)=\frac{9}{7}\left(\operatorname{coth}^{2} 1-1\right) \approx$ 0.9309 .

\section{Bounds with the weighted power mean of order -2}

In this section, we look for optimal bounds for means $K<L<M$ of the form $\sqrt{\frac{1-\alpha}{M^{2}}+\frac{\alpha}{K^{2}}}<$ $\frac{1}{L}<\sqrt{\frac{1-\beta}{M^{2}}+\frac{\beta}{K^{2}}}$ or, in terms of their Seiffert functions,

$$
\alpha<\frac{l^{2}-m^{2}}{k^{2}-m^{2}}<\beta .
$$

Theorem 7 The inequalities

$$
\sqrt{\frac{1-\alpha}{C e^{2}}+\frac{\alpha}{A^{2}}}<\frac{1}{M_{\sin }}<\sqrt{\frac{1-\beta}{C e^{2}}+\frac{\beta}{A^{2}}}
$$

hold if, and only if, $\alpha \leq \frac{16 \sin ^{2} 1-9}{7} \approx 0.3327$ and $\beta \geq \frac{1}{2}$.

Proof Taking formula (5) into account we should investigate the function

$$
h(z)=\frac{\sin ^{2} z-\frac{9 z^{2}}{\left(z^{2}+3\right)^{2}}}{z^{2}-\frac{9 z^{2}}{\left(z^{2}+3\right)^{2}}}=\frac{\left(z^{2}+3\right)^{2} \sin ^{2} z-9 z^{2}}{z^{4}\left(z^{2}+6\right)} .
$$

We shall show that $h$ decreases. We have

$$
\begin{aligned}
h^{\prime}(z) & =\frac{\left(z^{2}+3\right)\left(-z^{4}+27 z^{2}-36+\left(z^{2}+3\right)\left(z^{2}+6\right) z \sin 2 z+\left(z^{4}+9 z^{2}+36\right) \cos 2 z\right)}{z^{5}\left(z^{2}+6\right)^{2}} \\
& =: \frac{\left(z^{2}+3\right) s(z)}{z^{5}\left(z^{2}+6\right)^{2}} .
\end{aligned}
$$


From $\sin 2 x<2 x-(2 x)^{3} / 3 !+(2 x)^{5} / 5 !-(2 x)^{7} / 7 !+(2 x)^{9} / 9 !$ and $\cos 2 x<1-(2 x)^{2} / 2 !+$ $(2 x)^{4} / 4$ ! $-(2 x)^{6} / 6 !+(2 x)^{8} / 8$ ! we obtain

$$
\begin{aligned}
s(z)< & -z^{4}+27 z^{2}-36+\left(z^{2}+3\right)\left(z^{2}+6\right) z\left(2 z-\frac{(2 z)^{3}}{3 !}+\frac{(2 z)^{5}}{5 !}-\frac{(2 z)^{7}}{7 !}+\frac{(2 z)^{9}}{9 !}\right) \\
& +\left(z^{4}+9 z^{2}+36\right)\left(1-\frac{(2 z)^{2}}{2 !}+\frac{(2 z)^{4}}{4 !}-\frac{(2 z)^{6}}{6 !}+\frac{(2 z)^{8}}{8 !}\right) \\
= & \frac{2}{2835} z^{6}\left(2 z^{8}-9 z^{6}+45 z^{4}+999 z^{2}-6237\right)<0 .
\end{aligned}
$$

Thus $h^{\prime}(z)<0$. We complete the proof by noting that $\lim _{z \rightarrow 0} h(z)=1 / 2$.

Theorem 8 The inequalities

$$
\sqrt{\frac{1-\alpha}{C e^{2}}+\frac{\alpha}{A^{2}}}<\frac{1}{M_{\tanh }}<\sqrt{\frac{1-\beta}{C e^{2}}+\frac{\beta}{A^{2}}}
$$

hold if, and only if, $\alpha \leq 0$ and $\beta \geq \frac{16 \tanh ^{2} 1-9}{7} \approx 0.0401$.

Proof This time we investigate the function

$$
h(z)=\frac{\tanh ^{2} z-\frac{9 z^{2}}{\left(3+z^{2}\right)^{2}}}{z^{2}-\frac{9 z^{2}}{\left(3+z^{2}\right)^{2}}}=\frac{\left(z^{2}+3\right)^{2} \tanh ^{2} z-9 z^{2}}{z^{6}+6 z^{4}} .
$$

This function increases, because by Lemma 4

$$
\begin{aligned}
h^{\prime}(z) & =\frac{z^{2}+3}{2 z^{5}\left(z^{2}+6\right)^{2} \cosh ^{3} z}\left(\begin{array}{c}
4 z\left(z^{2}+3\right)\left(z^{2}+6\right) \sinh z \\
+\left(z^{4}+63 z^{2}+36\right) \cosh z \\
-\left(z^{4}-9 z^{2}+36\right) \cosh 3 z
\end{array}\right) \\
& >\frac{z^{2}+3}{2 z^{5}\left(z^{2}+6\right)^{2} \cosh ^{3} z}\left(\begin{array}{c}
4 z\left(z^{2}+3\right)\left(z^{2}+6\right)\left(z+\frac{z^{3}}{3 !}+\frac{z^{5}}{5 !}+\frac{z^{7}}{7 !}\right) \\
+\left(z^{4}+63 z^{2}+36\right)\left(1+\frac{z^{2}}{2 !}+\frac{z^{4}}{4 !}+\frac{z^{6}}{6 !}+\frac{z^{8}}{8 !}\right) \\
-\left(z^{4}-9 z^{2}+36\right)\left(1+\frac{(3 z)^{2}}{2 !}+\frac{(3 z)^{4}}{4 !}+\frac{(3 z)^{6}}{6 !}+\frac{3}{2} \times \frac{(3 z)^{8}}{8 !}\right)
\end{array}\right) \\
& =\frac{z\left(z^{2}+3\right)}{2\left(z^{2}+6\right)^{2} \cosh ^{3} z} \times \frac{-19617 z^{6}+99001 z^{4}-156324 z^{2}+258048}{80640}>0 .
\end{aligned}
$$

So the function $h$ assumes values between $\lim _{z \rightarrow 0} h(z)=0$ and $h(1)$.

\section{Bounds with varying arguments}

If $N$ is a mean, then the formula $N^{\{t\}}(x, y)=N\left(\frac{x+y}{2}+t \frac{x-y}{2}, \frac{x+y}{2}-t \frac{x-y}{2}\right)$ defines a homotopy between the arithmetic mean $\mathrm{A}=N^{\{0\}}$ and $N=N^{\{1\}}$. Therefore, if $\mathrm{A}<M<N$, it makes sense to ask what the optimal numbers $\alpha, \beta$ are satisfying $N^{\{\alpha\}}<M<N^{\{\beta\}}$. Theorem 6.1 from [19] gives a method for finding such numbers in terms of the Seiffert functions of the means involved. It says

Theorem 9 For a Seiffert function $k$, denote $\widehat{k}(z)=k(z) / z$. Let $M$ and $N$ be two means with Seiffert functions $m$ and $n$, respectively. Suppose that $\widehat{n}(z)$ is strictly monotone and let $p_{0}=\inf _{z} \frac{\widehat{n}^{-1}(\widehat{m}(z))}{z}$ and $q_{0}=\sup _{z} \frac{\widehat{n}^{-1}(\widehat{m}(z))}{z}$. 
If $A(x, y)<M(x, y)<N(x, y)$ for all $x \neq y$, then the inequalities

$$
N^{\{p\}}(x, y) \leq M(x, y) \leq N^{\{q\}}(x, y)
$$

hold if, and only if, $p \leq p_{0}$ and $q \geq q_{0}$.

If $N(x, y)<M(x, y)<A(x, y)$ for all $x \neq y$, then the inequalities

$$
N^{\{q\}}(x, y) \leq M(x, y) \leq N^{\{p\}}(x, y)
$$

hold if, and only if, $p \leq p_{0}$ and $q \geq q_{0}$.

In the case of $N=$ Ce we see that $\widehat{c e}(z)=\frac{3}{z^{2}+3}$ and $\widehat{c e}^{-1}(x)=\sqrt{3 x^{-1}-3}$.

Theorem 10 The inequalities

$$
\mathrm{Ce}\left(\frac{x+y}{2}+\alpha \frac{x-y}{2}, \frac{x+y}{2}-\alpha \frac{x-y}{2}\right)<M_{\sin }<\mathrm{Ce}\left(\frac{x+y}{2}+\beta \frac{x-y}{2}, \frac{x+y}{2}-\beta \frac{x-y}{2}\right)
$$

hold if, and only if, $\alpha \leq \sqrt{\frac{1}{2}} \approx 0.7071$ and $\beta \geq \sqrt{\frac{3}{\sin 1}-3} \approx 0.7518$.

Proof Using Theorem 9 we should find the range of the function

$$
h(z)=\frac{\sqrt{3 \frac{z}{\sin z}-3}}{z} .
$$

The monotonicity of the function $h^{2}$ follows from the proof of Theorem 1 , so evaluation of the values of $h$ at the endpoints completes the proof.

Theorem 11 The inequalities

$$
\mathrm{Ce}\left(\frac{x+y}{2}+\alpha \frac{x-y}{2}, \frac{x+y}{2}-\alpha \frac{x-y}{2}\right)<M_{\mathrm{tanh}}<\mathrm{Ce}\left(\frac{x+y}{2}+\beta \frac{x-y}{2}, \frac{x+y}{2}-\beta \frac{x-y}{2}\right)
$$

hold if, and only if, $\alpha \leq \sqrt{3 \operatorname{coth} 1-3} \approx 0.9691$ and $\beta \geq 1$.

Proof According to Theorem 9, we shall consider the function

$$
h(z)=\frac{\sqrt{3 \frac{z}{\tanh z}-3}}{z},
$$

but we found the range of its square in the proof of Theorem 2 .

\section{Tools and lemmas}

In this section, we place all the technical details needed to prove our main results.

Property 1 A function $f:(a, b) \rightarrow \mathbb{R}$ is convex if, and only if, for every $a<\theta<b$ its divided difference $\frac{f(x)-f(\theta)}{x-\theta}$ increases for $x \neq \theta$.

A simple consequence of Property 1 is

Property 2 If a function $f:(a, b) \rightarrow \mathbb{R}$ is convex and $\lim _{x \rightarrow a} f(x)=\Theta$, then the function $\frac{f(x)-\Theta}{x-a}$ increases.

Lemma 1 For all positive $x \neq y$ the inequality $M_{\tanh }(x, y)<\operatorname{Ce}(x, y)$ holds. 
Proof Using Seiffert's functions we have to proof that $h(z)=\tanh z-\frac{3 z}{3+z^{2}}>0$ for $0<$ $z<1$. Note that

$$
\begin{aligned}
\cosh z & =1+\frac{z^{2}}{2 !}+\frac{z^{4}}{4 !}+\frac{z^{6}}{6 !}+\frac{z^{8}}{8 !} \cdots \\
& <1+\frac{z^{2}}{2 !}+\frac{z^{4}}{4 !}\left(1+\frac{1}{2}+\frac{1}{4}+\cdots\right)=1+\frac{z^{2}}{2}+\frac{z^{4}}{12}
\end{aligned}
$$

This yields

$$
\begin{aligned}
h^{\prime}(z) & =\frac{1}{\cosh ^{2} z}-\frac{9-3 z^{2}}{\left(3+z^{2}\right)^{2}}>\frac{1}{\left(1+\frac{z^{2}}{2}+\frac{z^{4}}{12}\right)^{2}}-\frac{9-3 z^{2}}{\left(3+z^{2}\right)^{2}} \\
& =\frac{3 z^{4}\left(z^{6}+9 z^{4}+24 z^{2}+12\right)}{\left(3+z^{2}\right)^{2}\left(12+6 z^{2}+z^{4}\right)^{2}}>0
\end{aligned}
$$

which, combined with $h(0)=0$ completes the proof.

Lemma 2 (Lazarević [9]) Consider the functions $g_{u}:[0, \infty) \rightarrow \mathbb{R}$

$$
g_{u}(x)=\cosh ^{u} x \sinh x-x, \quad-1<u<0 .
$$

For $-1 / 3 \leq u<0$, the functions $g_{u}$ are positive. For $-1<u<-1 / 3$, there exists $x_{u}>0$, such that $g_{u}$ is negative in $\left(0, x_{u}\right)$ and positive in $\left(x_{u}, \infty\right)$.

Proof We have $g_{u}(0)=g_{u}^{\prime}(0)=0$ and

$$
g_{u}^{\prime \prime}(x)=u(u-1) \sinh x \cosh ^{u} x\left[\tanh ^{2} x+\frac{1+3 u}{u(u-1)}\right] .
$$

If $-1 / 3 \leq u<0$, we have $\frac{1+3 u}{u(u-1)} \geq 0$, so $g_{u}$ is convex thus positive. For $-1<u<-1 / 3$, the equation $\tanh ^{2} x+\frac{1+3 u}{u(u-1)}=0$ has exactly one solution $\xi_{u}$, so $g_{u}$ is concave and negative on $\left(0, \xi_{u}\right)$. Then it becomes convex and tends to infinity, thus assumes zero at exactly one point $x_{u}$.

The next lemma can be found in [1, Theorem 1.25].

Lemma 3 Suppose $f, g:(a, b) \rightarrow \mathbb{R}$ are differentiable with $g^{\prime}(x) \neq 0$ and such that $\lim _{x \rightarrow a} f(x)=\lim _{x \rightarrow a} g(x)=0$ or $\lim _{x \rightarrow b} f(x)=\lim _{x \rightarrow b} g(x)=0$. Then

1. if $\frac{f^{\prime}}{g^{\prime}}$ is increasing on $(a, b)$, then $\frac{f}{g}$ is increasing on $(a, b)$,

2. if $\frac{f^{\prime}}{g^{\prime}}$ is decreasing on $(a, b)$, then $\frac{f}{g}$ is decreasing on $(a, b)$.

Lemma 4 For $0<x<1$, the following inequalities hold

(a) $\sinh 3 x<3 x+\frac{(3 x)^{3}}{3 !}+\frac{(3 x)^{5}}{5 !}+\frac{(3 x)^{7}}{7 !}+2 \frac{(3 x)^{9}}{9 !}$,

(b) $\sinh 2 x<2 x+\frac{(2 x)^{3}}{3 !}+\frac{(2 x)^{5}}{5 !}+2 \frac{(2 x)^{7}}{7 !}$,

(c) $\cosh 3 x<1+\frac{(3 x)^{2}}{2 !}+\frac{(3 x)^{4}}{4 !}+\frac{(3 x)^{6}}{6 !}+\frac{3}{2} \times \frac{(3 x)^{8}}{8 !}$.

Proof a)

$$
\sinh (3 x)-3 x-\frac{(3 x)^{3}}{3 !}-\frac{(3 x)^{5}}{5 !}-\frac{(3 x)^{7}}{7 !}-\frac{(3 x)^{9}}{9 !}
$$




$$
\begin{aligned}
= & \frac{(3 x)^{11}}{11 !}+\frac{(3 x)^{13}}{13 !}+\cdots<\frac{(3 x)^{9}}{9 !}\left(\frac{3^{2}}{10 \cdot 11}+\frac{3^{4}}{10 \cdot 11 \cdot 12 \cdot 13}+\cdots\right) \\
& <\frac{(3 x)^{9}}{9 !}
\end{aligned}
$$

Other proofs are similar.

\section{Declarations}

Conflict of interest The authors declare that they have no conflict of interest.

Open Access This article is licensed under a Creative Commons Attribution 4.0 International License, which permits use, sharing, adaptation, distribution and reproduction in any medium or format, as long as you give appropriate credit to the original author(s) and the source, provide a link to the Creative Commons licence, and indicate if changes were made. The images or other third party material in this article are included in the article's Creative Commons licence, unless indicated otherwise in a credit line to the material. If material is not included in the article's Creative Commons licence and your intended use is not permitted by statutory regulation or exceeds the permitted use, you will need to obtain permission directly from the copyright holder. To view a copy of this licence, visit http://creativecommons.org/licenses/by/4.0/.

\section{References}

1. Anderson, G.D., Vamanamurthy, M.K., Vuorinen, M.K.: Conformal invariants, inequalities, and quasiconformal maps. Canadian Mathematical Society Series of Monographs and Advanced Texts. Wiley, New York (1997). With 1 IBM-PC floppy disk (3.5 inch; HD), A Wiley-Interscience Publication

2. Chu, H.H., Zhao, T.H., Chu, Y.M.: Sharp bounds for the Toader mean of order 3 in terms of arithmetic, quadratic and contraharmonic means. Math. Slovaca 70(5), 1097-1112 (2020). https://doi.org/10.1515/ ms-2017-0417

3. Chu, Y.M., Long, B.Y.: Bounds of the Neuman-Sándor mean using power and identric means. Abstr. Appl. Anal. 6, Art. ID 832591 (2013). https://doi.org/10.1155/2013/832591

4. Chu, Y.M., Yang, Z.H., Wu, L.M.: Sharp power mean bounds for Sándor mean. Abstr. Appl. Anal. 5, Art. ID 172867 (2015). https://doi.org/10.1155/2015/172867

5. He, X.H., Qian, W.M., Xu, H.Z., Chu, Y.M.: Sharp power mean bounds for two Sándor-Yang means. Rev. R. Acad. Cienc. Exactas Fís. Nat. Ser. A Mat. RACSAM 113(3), 2627-2638 (2019). https://doi.org/ 10.1007/s13398-019-00643-2

6. He, Z.Y., Wang, M.K., Jiang, Y.P., Chu, Y.M.: Bounds for the perimeter of an ellipse in terms of power means. J. Math. Inequal. 14(3), 887-899 (2020). https://doi.org/10.7153/jmi-2020-14-58

7. Hua, Y., Qi, F.: The best bounds for Toader mean in terms of the centroidal and arithmetic means. Filomat 28(4), 775-780 (2014). https://doi.org/10.2298/FIL1404775H

8. Hua, Y., Qi, F.: A double inequality for bounding Toader mean by the centroidal mean. Proc. Indian Acad. Sci. Math. Sci. 124(4), 527-531 (2014). https://doi.org/10.1007/s12044-014-0183-6

9. Lazarević, I.: Certain inequalities with hyperbolic functions. Univ. Beograd. Publ. Elektrotehn. Fak. Ser. Mat. Fiz. No. 159-170, 41-48 (1966)

10. Li, Y.M., Long, B.Y., Chu, Y.M.: Sharp bounds for the Neuman-Sándor mean in terms of generalized logarithmic mean. J. Math. Inequal. 6(4), 567-577 (2012). https://doi.org/10.7153/jmi-06-54

11. Nowicka, M., Witkowski, A.: Optimal bounds for the sine and hyperbolic tangent means. Punjab Univ. J. Math. (Lahore) 52(5), 77-88 (2020)

12. Nowicka, M., Witkowski, A.: Optimal bounds for the sine and hyperbolic tangent means II. J. Appl. Anal. 27(1) (2021). https://doi.org/10.1515/jaa-2020-2032 (to appear)

13. Qian, W.M., Chu, Y.M.: Best possible bounds for Yang mean using generalized logarithmic mean. Math. Probl. Eng. 7, Art. ID 8901258 (2016). https://doi.org/10.1155/2016/8901258

14. Qian, W.M., He, Z.Y., Zhang, H.W., Chu, Y.M.: Sharp bounds for Neuman means in terms of twoparameter contraharmonic and arithmetic mean. J. Inequal. Appl. 13, Paper No. 168 (2019). https://doi. org/10.1186/s13660-019-2124-5 
15. Qian, W.M., Yang, Y.Y., Zhang, H.W., Chu, Y.M.: Optimal two-parameter geometric and arithmetic mean bounds for the Sándor-Yang mean. J. Inequal. Appl. 12, Paper No. 287 (2019). https://doi.org/10.1186/ s13660-019-2245-X

16. Qian, W.M., Zhang, X.H., Chu, Y.M.: Sharp bounds for the Toader-Qi mean in terms of harmonic and geometric means. J. Math. Inequal. 11(1), 121-127 (2017). https://doi.org/10.7153/jmi-11-11

17. Song, Y.Q., Qian, W.M., Chu, Y.M.: Optimal bounds for Neuman mean using arithmetic and centroidal means. J. Funct. Spaces 7, Art. ID 5131907 (2016). https://doi.org/10.1155/2016/5131907

18. Wang, B., Luo, C.L., Li, S.H., Chu, Y.M.: Sharp one-parameter geometric and quadratic means bounds for the Sándor-Yang means. Rev. R. Acad. Cienc. Exactas Fís. Nat. Ser. A Mat. RACSAM 114(1), Paper No. 7, 10 (2020). https://doi.org/10.1007/s13398-019-00734-0

19. Witkowski, A.: On Seiffert-like means. J. Math. Inequal. 9(4), 1071-1092 (2015). https://doi.org/10. 7153/jmi-09-83

20. Xia, W., Chu, Y.: Optimal inequalities between Neuman-Sándor, centroidal and harmonic means. J. Math. Inequal. 7(4), 593-600 (2013). https://doi.org/10.7153/jmi-07-56

21. Xu, H.Z., Chu, Y.M., Qian, W.M.: Sharp bounds for the Sándor-Yang means in terms of arithmetic and contra-harmonic means. J. Inequal. Appl. 13, Paper No. 127 (2018). https://doi.org/10.1186/s13660-0181719-6

Publisher's Note Springer Nature remains neutral with regard to jurisdictional claims in published maps and institutional affiliations. 